\title{
FRUGIVORIA POR AVES EM ÁREA DE CERRADO NO MUNICÍPIO DE UBERLÂNDIA, MINAS GERAIS ${ }^{1}$
}

\author{
Gustavo Bernardino Malacco da Silva² e Fernando Pedroni ${ }^{3}$
}

\begin{abstract}
RESUMO - O consumo de frutos carnosos por vertebrados é um fenômeno importante nos trópicos, com destaque para aves e mamíferos que apresentam altas proporções de espécies frugívoras. O objetivo do estudo foi observar as principais aves frugívoras em área de Cerrado na reserva do Clube Caça e Pesca Itororó, no Município de Uberlândia, MG, durante o período de novembro de 2000 a outubro de 2001. Foram registradas 90 interações, envolvendo 22 espécies de plantas e 33 espécies de aves. Foi encontrado padrão assimétrico nas interações, no qual poucas espécies são responsáveis pela maioria das interações. As principais aves observadas consumindo frutos são de pequeno porte e onívoras. Interações frugívoras com aves de grande porte, como das famílias Cracidae e Ramphastidae, consideradas potenciais dispersores de sementes, não foram registradas. O estudo demonstrou a importância das espécies vegetais Ouratea spectabilis e Miconia theizans nas interações de frugivoria, além do destaque do gênero Elaenia no consumo de frutos e dispersão de sementes.
\end{abstract}

Palavras-chave: Frutívora; Cerrado; Aves.

\section{FRUGIVORY BY BIRDS IN CERRADO IN THE CITY OF UBERLÂNDIA, MINAS GERAIS}

\begin{abstract}
The consumption of fleshy fruits by vertebrates is an important phenomenon in the tropics, especially birds and mammals that have high proportions of frugivorous species. The aim of this study was to observe the main frugivorous birds in the reserve Cerrado area of the Clube Caça e Pesca Itororó, Uberlândia, MG, during the period extending from November 2000 to October 2001. We recorded 90 interactions involving 22 species of plants and 33 species of birds. Asymmetrical pattern was found in the interactions in which few species are responsible for most interactions. The main birds observed consuming fruits are small size and omnivorous. Frugivorous interactions with large sized birds, such as the Cracidae and Ramphastidae families, considered as potential seed dispersers, were not recorded. The study demonstrated the importance of the plant species Ouratea spectabilis and Miconia theizans in frugivory interactions, and highlighted the genus EIaenia as a fruit consumer and seed disperser.
\end{abstract}

Keywords: Frugivory; Cerrado; Birds.

\section{INTRODUÇÃO}

Nos trópicos, a maioria das plantas depende de animais para transporte de pólen e para dispersão de suas sementes. Em troca desse serviço, as plantas fornecem recursos florais como pólen, néctar e óleo, além de frutos com polpas e arilos suculentos ou sementes nutritivas (MORELLATO; LEITÃO-FILHO, 1992). Nas florestas tropicais, a forma de dispersão de sementes mais comum é a zoocoria, em que espécies vegetais apresentam frutos adaptados à dispersão por animais (MORELLATO; LEITÃO-FILHO, 1992; JORDANO, 2000; ALMEIDA-NETO et al., 2008). Em florestas de galeria e Cerrados sensu stricto do Brasil Central e Centro Austral, a zoocoria é o principal modo de dispersão de sementes encontrado, variando entre 51,1\% e 89,4\% do total de espécies (GOTTSBERGER; SILLBERBAUER-GOTTSBERGER, 1983; MOTTAJÚNIOR; LOMBARDI, 2002; VIEIRA et al., 2002). As

\footnotetext{
${ }^{1}$ Recebido em 24.04.2013 aceito para publicação em 09.04.2014.

${ }^{2}$ Associação para Gestão Socioambiental do Triângulo Mineiro (Angá), Uberlândia, MG, Brasil. E-mail: <malacco@gmail.com>. ${ }^{3}$ Universidade Federal de Mato Grosso, Instituto de Ciências Biológicas e da Saúde, MT, Brasil. E-mail: < fpedroni@cpd.ufmt.br>.
} 
plantas zoocóricas concentram os picos de frutificação no início da estação chuvosa (GOTTSBERGER; SILLBERBAUER-GOTTSBERGER, 1983; SILVA et al., 2009; PIRANI et al., 2009), aumentando as chances de germinação de sementes dispersas e crescimento de plântulas (FELFILI et al., 1999) durante a estação favorável.

As aves frugívoras dispersoras de sementes podem desempenhar papel fundamental na recuperação da vegetação em áreas alteradas pela interferência antrópica ou por fenômenos naturais (WUNDERLE JÚNIOR, 1997; GARCIA et al., 2009). Dessa forma, as interações plantafrugívoros são importantes nas complexas comunidades tropicais, e a modificação ou perdas dessas interações podem ter profundas implicações para conservação (SILVA; TABARELLI, 2000; SILVAet al., 2002). Algumas espécies de plantas são importantes para manutenção de populações de animais frugívoros, principalmente quando frutificam durante períodos de escassez de frutos (TERBORGH, 1986; PERES, 2000). Este estudo objetivou: 1) analisar as interações entre aves frugívoras e plantas; e 2) apresentar a fenologia de frutificação das espécies utilizadas pelas aves em área de Cerrado do Triângulo Mineiro, Minas Gerais.

\section{MATERIAL E MÉTODOS}

Área de estudo - O estudo foi realizado na Reserva do Clube Caça e Pesca Itororó (CCPIU) (1857’S e 48¹2’W), Município de Uberlândia, Triângulo Mineiro, MG. A reserva possui 600 ha, localizada a cerca de $8 \mathrm{~km}$ do centro urbano. A vegetação abrange vários tipos fisionômicos, incluindo Cerrado sentido restrito, veredas e florestas paludosas (APOLLINÁRIO; SCHIAVINI, 1992). As médias anuais de precipitação e temperatura são, respectivamente, $1.550 \mathrm{~mm}$ e $22^{\circ} \mathrm{C}$ (ROSA et al., 1991).

Observações das interações - As observações da fenologia de frutificação e consumo de frutos ocorreram entre novembro de 2000 e outubro de 2001, sendo realizadas de três a cinco visitas mensais (49 visitas no total), totalizando 196 h de observação. Foi percorrido um transecto de aproximadamente $3 \mathrm{~km}$ ao longo da transição entre o Cerrado sentido restrito e a vereda. As observações foram feitas com binóculos $(10 \times 42 \mathrm{~mm})$ desde o amanhecer até as $10 \mathrm{~h}$. As identificações das aves seguiram o Comitê Brasileiro de Registros Ornitológicos (2014). Em relação às guildas de alimentação, as aves foram classificadas em insetívoras, onívoras, frugívoras e granívoras, de acordo com MottaJúnior (1990), Sick (1997) e Marini e Cavalcanti (1998). Para definição do peso corporal das aves, seguiu-se Cavalcanti e Marini (1993), bem como informações oriundas de capturas realizadas na reserva pelos autores.

Durante as observações, ao longo do transecto, foram feitos registros de alimentação ("feeding-bouts"). Sempre que um (ou mais) indivíduo de determinada espécie de ave foi avistado consumindo frutos de uma planta, foi anotado um registro de alimentação. Se um (ou mais) desses indivíduos se deslocou para outra planta e consumiu frutos, foi anotado um novo registro de alimentação. Devido à dificuldade de identificação em campo, espécies do gênero Elaenia e fêmeas do gênero Sporophila foram consideradas nos complexos Elaenia spp. e Sporophila spp. Foi registrado o tempo de permanência da ave durante o registro de alimentação e o tipo de comportamento de retirada (MOERMOND; DENSLOW, 1985) e de consumo do fruto (SCHUPP, 1993). Para identificação das plantas utilizadas pelas aves frugívoras, foram coletadas e herborizadas amostras em estado reprodutivo, para comparação com exemplares do herbário da Universidade Federal de Uberlândia.

Análise estatística - Para análise das relações na comunidade, os registros de alimentação de cada espécie de ave sobre uma espécie vegetal foram considerados uma interação, sendo as interações consideradas binárias. As interações foram arranjadas numa matriz de espécies de aves por espécies de plantas, a partir da qual foram calculados o número de interações e o índice de importância das espécies de plantas e aves. O índice foi desenvolvido por Murray (2000 apud SILVA et al., 2002) e é dado pela equação:

$$
I j=\sum_{i=1}^{S}\left(\frac{C i, j / T i}{S}\right)
$$

em que:

$\mathrm{T}_{i}=$ número total de espécies de aves que forrageiam uma espécie de planta $i ; S=$ total de espécies de plantas incluídas na amostra; e $C_{i, j}=1$ se a espécie de ave $j$ consome frutos da espécie de planta $i$ ou 0 se isso não ocorre.

Valores de $\mathrm{I}_{j}$ podem variar de 0.0 para aves que não consomem frutos até 1.0 para aves que consomem frutos de todas as espécies da comunidade. A mesma equação pode ser usada para estimar a importância relativa de diferentes espécies de plantas na dieta das 
aves $\left(\mathrm{I}_{j}\right)$; nesse caso: $\mathrm{T}_{i}=$ número total de espécies de plantas que a espécie de ave $i$ forrageia; $S=$ número total de espécies de aves incluídas na amostra; e $\mathrm{C}_{i, j}$ = 1 se a espécie de planta $j$ é incluída na dieta da espécie de ave $i$ ou 0 se isso não ocorre.

Foram utilizadas correlações de Spearman $\left(\mathrm{R}_{\mathrm{s}}\right)$ para avaliar a relação entre o número mensal de interações observadas e o de espécies de plantas frutificando no mês.

\section{RESULTADOS}

Plantas - Foram observadas interações em 21 espécies de plantas, distribuídas em 14 famílias, sendo as mais ricas Myrtaceae ( $\mathrm{n}=6 \mathrm{spp}$.) e Melastomataceae $(n=3)$. As espécies vegetais observadas variaram quanto ao hábitat de ocorrência, hábito e tipo e coloração dos frutos (Tabela 1). A frutificação ocorreu durante todo o ano, com pico no número de espécies com frutos maduros durante a estação chuvosa (outubro-abril), principalmente entre outubro e janeiro. Os meses com maior número de espécies com frutos maduros foram janeiro $(n=11)$ e dezembro $(n=13)$.

Aves consumindo frutos - Considerando os complexos de Elaenia spp. e Sporophila spp., foram registradas interações entre 33 espécies de aves, totalizando 90 interações (de 693 interações possíveis), oriundos de 143 registros de alimentação (Tabela 2). As plantas com maior número de interações pertencem às famílias Myrtaceae e Melastomataceae (n = 21, em ambas) (Tabela 1). As plantas com maior índice de importância foram Miconia theizans (Bonpl.) Cogn. (0,2173; 15 espécies de aves) $e$ Ouratea spectabilis (Mart.) Engl. (0,1523; 10 espécies de aves) (Figura 1).

Indivíduos de espécies com frutos do tipo baga apresentaram maior número de interações com aves do que indivíduos com outros tipos de frutos $(n=66$, $73,3 \%)$ e espécies com frutos vermelhos ( $n=24,26,6 \%)$ e atropurpúreos ( $\mathrm{n}=35,36,9 \%$ ) foram mais consumidas em relação a espécies com frutos de outras cores.

As famílias de aves com maior número de interações foram Tyrannidae ( $n=31,8$ espécies de aves) e Thraupidae ( $n=32,8$ spp.), totalizando $70 \%$ dos registros. Apenas seis interações (6,7\%) foram registradas para Não Passeriformes.

As aves com maior índice de importância foram Tangara palmarum (0,1381; nove espécies de plantas) e Turdus leucomelas (0,08651, 5 spp.), mas destacou-se
Elaenia spp. (0,2373, 15 spp.) (Figura 2). Cinco espécies de aves (Elaenia spp., Tangara palmarum, Neothraupis fasciata, Tyrannus melancholicus e Tangara cayana) foram responsáveis por $50 \%(n=45)$ das interações.

Vinte e nove espécies foram consideradas potencialmente dispersoras de sementes. Entre essas, a maioria apresentou dieta onívora $(n=17,51,5 \%)$, representando 57,8\% das interações observadas, enquanto as espécies frugívoras foram responsáveis por 23,3\% das interações. A segunda guilda alimentar mais frequente foi a insetívora ( $\mathrm{n}=7,21,2 \%)$ (Tabela 1$)$.

Foi observado maior número de interações entre aves e plantas e também de espécies de aves forrageando plantas nos meses de julho ( $\mathrm{n}=17 \mathrm{e} \mathrm{n}=12$, respectivamente) e setembro ( $n=16$ e $n=12$, respectivamente) (Figura 3 ). Mas durante a estação chuvosa, entre os meses de outubro a abril, foi observado maior número de interações (n = 70, 61,4\%) em relação à estação seca.

O número mensal de espécies em frutificação foi correlacionado positivamente com o número de interações de aves $\left(R_{s}=0,5868, n=12, p=0,045\right)$, mas não com o número de espécies de aves forrageando $\left(\mathrm{R}_{\mathrm{s}}=0,5406, \mathrm{n}=12, \mathrm{p}=0,070\right)$.

O tempo de permanência para alimentação em uma planta variou entre 1 s e $9,16 \min (\bar{x}=1,72 \pm 1,79$ min). As aves Não Passeriformes foram o grupo com maior tempo de visita ( $\bar{x}=5,78 \pm 2,81 \mathrm{~min})$, com destaque para Psittaciformes ( = 7,35 $\pm 1,95 \mathrm{~min}$ ). Representantes da família Tyrannidae apresentaram tempo médio de visita de 0,88 \pm 0,42 min e entre os Thraupidae 1,31 $\pm 0,62 \mathrm{~min}$. A maioria dos registros de alimentação durou menos de $2 \min (n=130,90,9 \%)$.

A maioria das aves observadas consumindo frutos possui menos de $50 \mathrm{~g}$ de massa corpórea (20 espécies) e representou $77,7 \%$ dos registros de alimentação. O comportamento de captura no poleiro foi o mais frequente (69,9\%), sendo observado principalmente entre Thraupidae (31,5\%), enquanto aves da família Tyrannidae foram observadas capturando frutos em voo (29,4\%).

A maioria das aves foi observada engolindo frutos (65,1\%) com ou sem mandibulação, e as aves Tyrannidae engoliram todos os frutos que conseguiam. No entanto, representantes de Thraupidae foram observados derrubando os frutos sob a planta-mãe ou apenas bicando e abandonando os frutos na planta-mãe, em $67,4 \%$ dos registros. Os Psittacidae predaram os frutos em $100 \%$ dos registros.

Revista Árvore, Viçosa-MG, v.38, n.3, p.433-442, 2014 
Tabela 1 - Espécies vegetais com frutos consumidos por aves na Reserva do Clube Caça e Pesca Itororó, Uberlândia, MG. Table 1 - Plant species with fruits consumed by birds in the Clube Caça e Pesca Itororó Reserve, Uberlândia, MG.

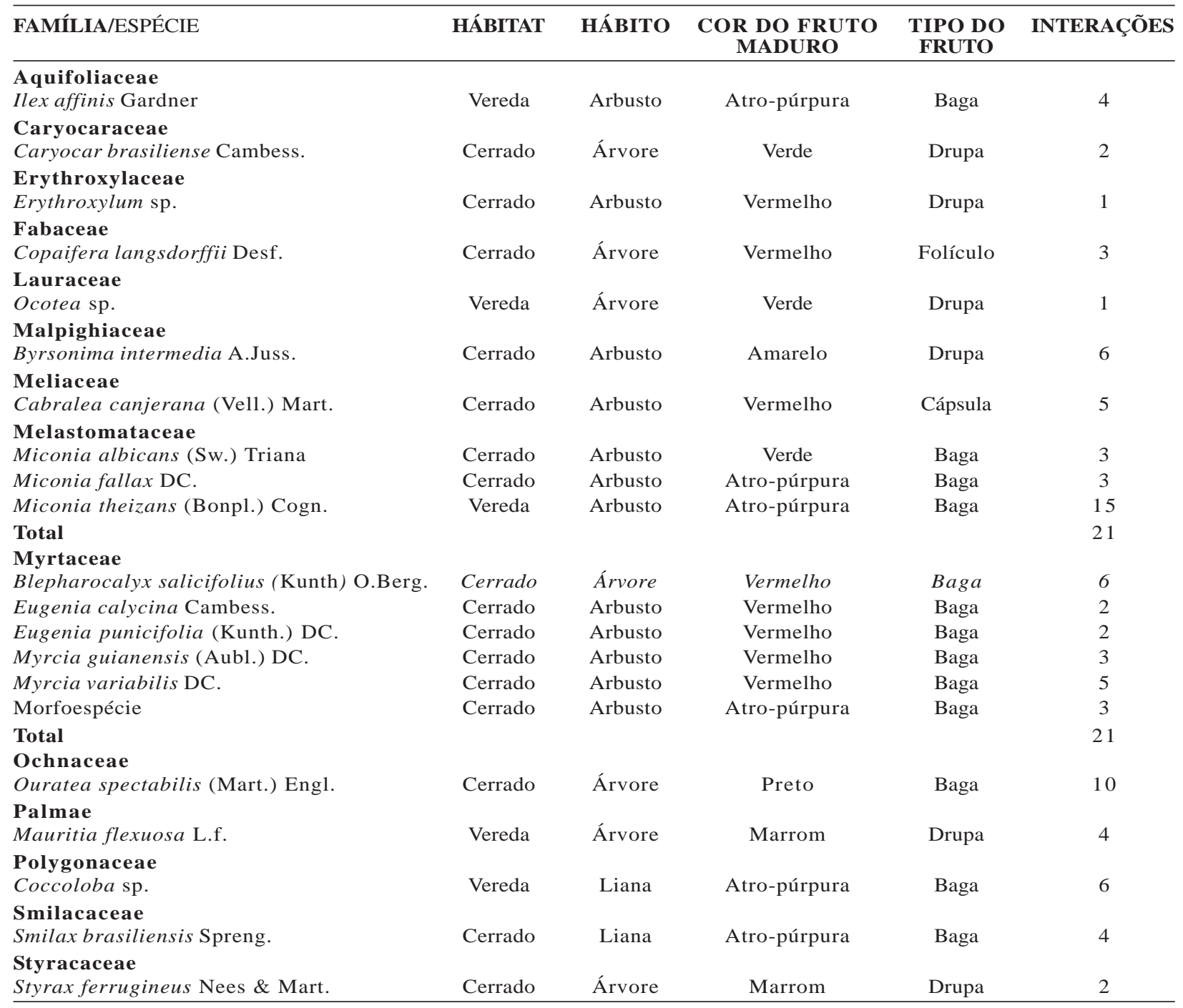

\section{DISCUSSÃO}

Em número de espécies de plantas, Myrtaceae foi mais visitada pelas aves. Essa família pode apresentar elevada riqueza de espécies, seja no Cerrado (PIRANI et al., 2009), sejana MataAtlântica(ATHIÊ; DIAS, 2012), sendo importante no fornecimento de frutos comestíveis para aves e mamíferos, uma vez que as suas espécies, na grande maioria, estavam adaptadas à dispersão por animais (GRESSLER et al., 2006).

Melastomataceae, no entanto, apresentou maior número de interações. A importância dessa família como recurso para aves nos neotrópicos já é conhecida (GALETTI;
STOTZ, 1996; LOISELLE;BLAKE, 1999;FADINI;MARCO JR., 2004; PARRINI; PACHECO, 2011). Silva et al. (2002), em estudo na Floresta Atlântica, destacaram a importância das famílias Melastomataceae e Myrtaceae para manutenção de frugívoros.

O predomínio de vermelho e de cores escuras entre os frutos consumidos pelas aves na reserva do Clube Caça e Pesca também foi registrado em áreas tropicais por Wheelwright e Janson (1985) e Faustino e Machado (2006), indicando que essas cores são particularmente atraentes para as aves. 


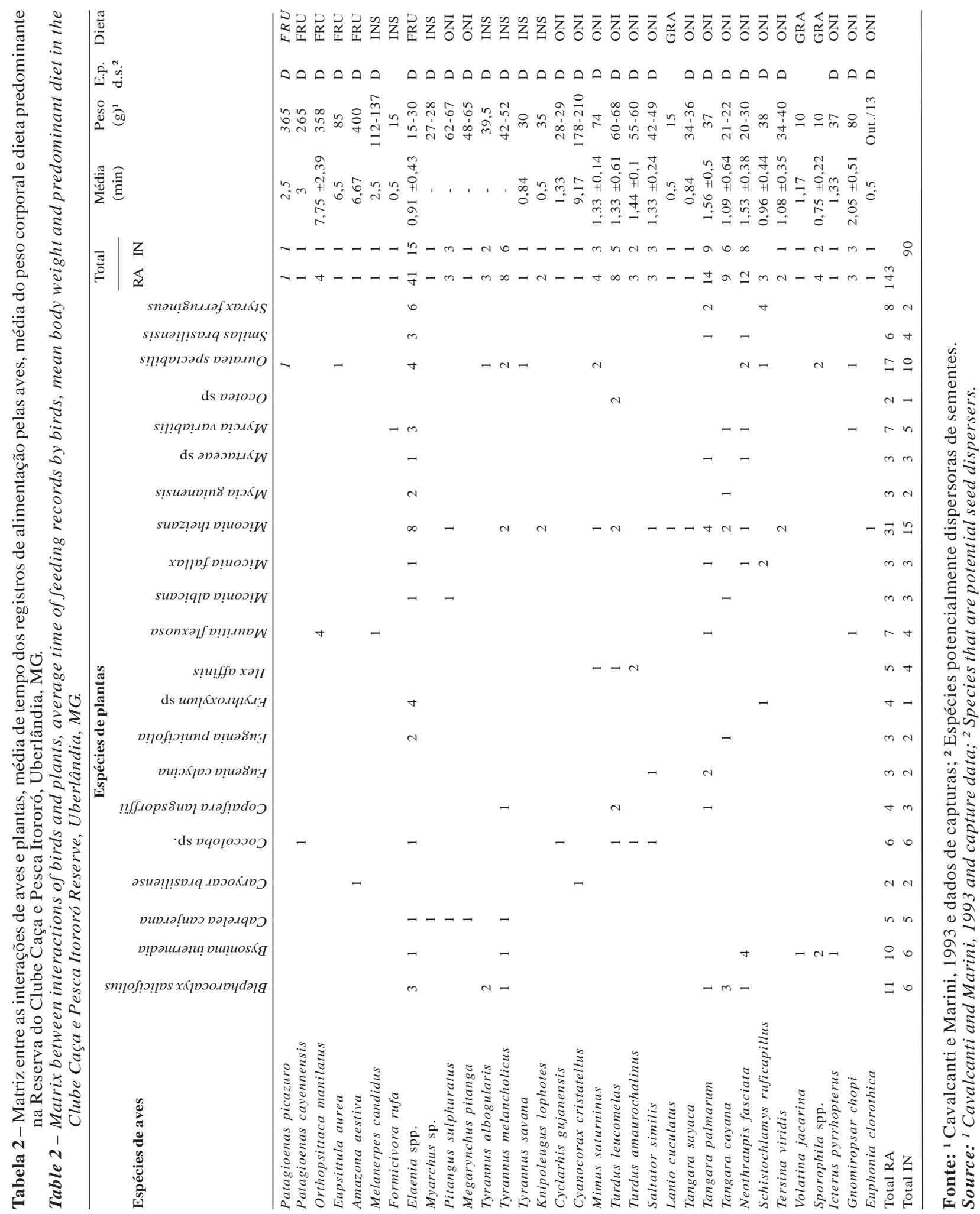

Revista Árvore, Viçosa-MG, v.38, n.3, p.433-442, 2014 


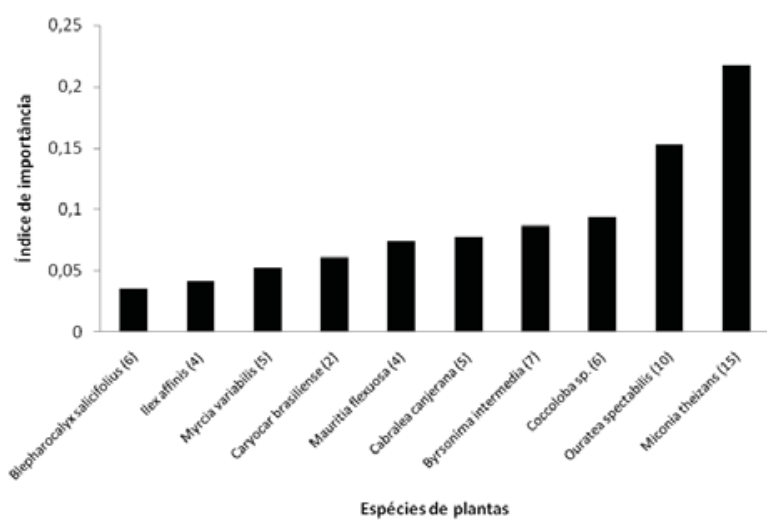

Figura 1 - Os 10 maiores índices de importância para plantas na Reserva do Clube Caça e Pesca Itororó, Uberlândia, MG. O número de interações de cada espécie é dado em parênteses.

Figure 1 - The 10 highest importance indexes for plants in Clube Caça e Pesca Itororó Reserve, Uberlândia, MG. The number of interactions of each species is given in parentheses.

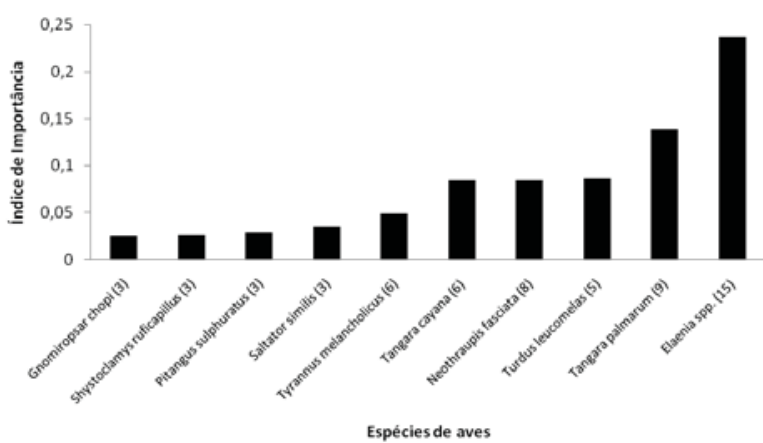

Figura 2 - Os 10 maiores índices de importância para aves na Reserva do Clube Caça e Pesca Itororó, Uberlândia, MG. O número de interações por espécie é dado em parênteses.

Figure 2 - The 10 highest importance indexes for birds in Clube Caça e Pesca Itororó Reserve, Uberlândia, MG. The number of interactions per species is given in parentheses.

Espécies zoocóricas tendem a concentrar a produção dos frutos durante o período úmido e quente (GOTTSBERGER; SILLBERBAUER-GOTTSBERGER, 1983; MANTOVANI; MARTINS, 1988; PIRANI et al., 2009), e a maturação dos frutos nesse período deve estar relacionada a condições mais propícias para germinação(MORELLATO; LEITÃO-FILHO, 1992), maior probabilidade de receber radiação solar e de crescimento das plântulas devido à umidade (JANZEN, 1967). Com a dispersão no início da estação chuvosa, as sementes

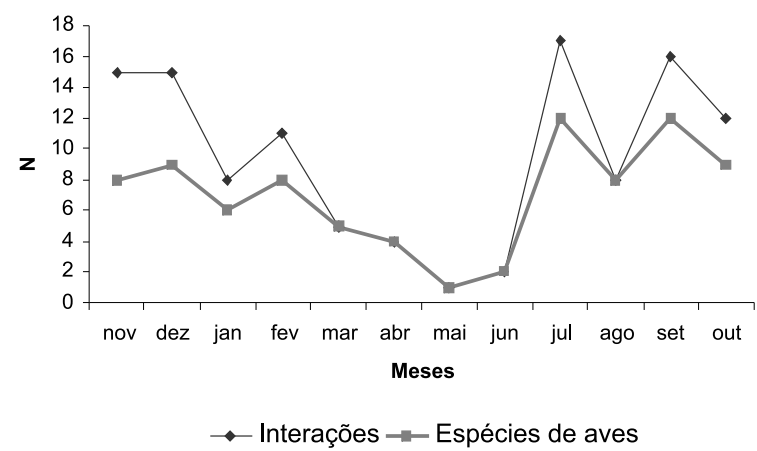

Figura 3 - Número mensal de interações $(\mathrm{n}=114)$ e número de espécies de aves $(n=33)$ observadas, alimentando-se de frutos na Reserva do Clube Caça e Pesca Itororó, Uberlândia, MG.

Figure 3 - Monthly number of interactions $(n=114)$ and number of bird species observed $(n=33)$ feeding on fruits in Clube Caça e Pesca Itororó Reserve, Uberlândia, $M G$.

teriam todo um período favorável para desenvolver o sistema radicular antes da próxima estação seca. Para Batalha et al. (1997), os frutos carnosos são atraentes por maior período na estação chuvosa, época de maior calor e umidade, propícia para a dispersão zoocórica.

A abundância dos dispersores representa fator final que pode influenciar a frutificação (GOTTSBERGER; SILLBERBAUER-GOTTSBERGER, 1983; PEDRONI et al., 2002), a qual pode estar ajustada ao período reprodutivo das aves ou à chegada de espécies migratórias (FOSTER, 1987). A chegada de populações migratórias no Cerrado ocorre na transição da estação seca para chuvosa, o que coincide com a frutificação da maioria das espécies. Muitas aves migratórias utilizam frutos, que representam recurso energeticamente rico para alimentar seus filhotes, como foi observado por Jesus e Monteiro-Filho (2007).

Na Reserva do Clube Caça e Pesca, foram registradas 90 interações, número semelhante ao encontrado em área de Mata Atlântica por Fadini e Marco Jr. (2004) e por Fonseca e Antunes (2007). Contabilizando apenas espécies vegetais nativas, as espécies Miconia theizans (Melastomataceae) e Ouratea spectabilis (Ochnaceae) foram importantes recursos alimentares para aves na Reserva do Clube Caça e Pesca.

$M$. theizans frutifica durante quase todo o ano, sendo importante fonte de alimento para aves em épocas de escassez de frutos, além de servir de poleiro para dispersão de outras espécies de plantas. Amâncio e 
Melo (2008) observaram alto número de visitas por aves frugívoras em estudo com $M$. theizans. $O$. spectabilis frutifica durante a estação chuvosa, sendo visitada por pelo menos 20 espécies de aves (G. MALACCO e colaboradores, dados inéditos). Como O. spectabilis é uma das espécies de plantas mais abundantes no CCPIU, assim como em outras áreas de Cerrado (CARVALHO et al., 2008; RIBEIRO et al., 2012), certamente essa espécie deve desempenhar papel importante na manutenção de aves frugívoras no Cerrado.

O padrão assimétrico das interações, com poucas espécies de aves e plantas respondendo pela maioria das interações, também foi registrado em outros trabalhos (SILVA et al., 2002; FADINI; MARCO JR., 2004; FONSECA; ANTUNES, 2007).A extinção local de qualquer uma das espécies, a não ser que seja substituída por outra, poderá significar alteração nos padrões de remoção e deposição de sementes, recrutamento das plantas e, ao longo do tempo, influenciar a estrutura da comunidade de plantas (LOISELLE; BLAKE, 2002). No CCPIU, as principais aves observadas consumindo frutos são generalistas (onívoras), e Wutherich et al. (2001), Fadini e Marco Jr. (2004) e Fonseca e Antunes (2007) também registraram onívoros como principais consumidores de frutos, indicando que espécies generalistas podem ser importantes na manutenção de áreas de Cerrado.

Registros de interações de frugívoros de grande porte como potenciais dispersores, a exemplo de representantes das Famílias Cracidae e Ramphastidae, foram inexistentes durante o estudo. A fragmentação de hábitat e a pressão de caça ocorridas na área de estudo podem ser fatores que contribuíram para que grandes frugívoros não tenham sido observados na reserva.

Em áreas alteradas de Cerrado (AMÂNCIO; MELO, 2008) e de Mata Atlântica (ATHIÊ; DIAS, 2012), Thraupidae e Tyrannidae representam as aves com maior número de interações de consumo de frutos. Entretanto, em áreas preservadas, famílias predominantemente frugívoras, como Cotingidae, Pipridae e Ramphastidae em conjunto com Thraupidae, contribuem com maior número de interações (SILVA et al., 2002; FADINI; MARCO JR., 2004). Segundo Snow e Snow (1971), as famílias Thraupidae e Tyrannidae podem ter importante papel na dispersão de sementes de espécies de plantas em áreas perturbadas nos neotrópicos. Rodrigues (1995) e Silva et al. (2002) também sugeriram esse padrão para a Floresta Atlântica. Portanto, essas famílias podem contribuir na recuperação e manutenção de processos ecológicos na Reserva.

As cinco espécies do gênero Elaenia apresentaram alto índice de importância no CCPIU. Dessas, com exceção de E. flavogaster e E. obscura, que são consideradas residentes (SICK, 1997 - observação pessoal), as demais Elaenia cristata, E. chiriquensis e E. spectabilis podem ser consideradas migrantes no CCPIU (SICK, 1997 observação pessoal). Essas três espécies, juntamente com outras espécies migratórias, influenciam o padrão de frutificação e dispersão de espécies zoocóricas, especialmente durante a estação chuvosa. A importância de espécies migrantes na dispersão também foi destacada em outros locais (GALETTI; PIZO, 1996; JESUS; MONTEIRO-FILHO, 2007).

A maioria das interações ocorreu na estação chuvosa ( $\mathrm{n}=70,61,4 \%)$, provavelmente devido ao maior número de espécies frutificando e à chegada de espécies de aves migratórias. Durante a estação seca, em julho ocorreu maior número de interações $(n=17)$ e de espécies de aves forrageando $(n=12)$, o que resultou da frutificação de duas espécies de plantas (Miconia theizans e Coccoloba sp.), que frutificam em época de escassez de frutos para a comunidade.

O principal comportamento de captura dos frutos foi o de poleiro, também verificado por Amâncio e Melo (2008) e Borges e Melo (2012). Mas a família Tyrannidae apresentou comportamento de captura dos frutos em voo, provavelmente pelo fato de essa família estar adaptada a capturar insetos em voo.

A duração de 90,9\% dos registros de alimentação foi inferior a $2 \mathrm{~min}$, o que, segundo Jordano e Schupp (2000), pode ser considerado tempo de permanência curto. Visitas curtas diminuem a probabilidade de as sementes ingeridas serem eliminadas embaixo da plantamãe, prevenindo a defecação ou regurgitação das sementes sob a planta-mãe (PRATT; STILES, 1983), aumentando a eficiência das aves como dispersoras (SCHUPP, 1993). Na maioria dos registros de alimentação feitos no CCPIU, os frutos foram engolidos (65,7\%), o que somado ao baixo tempo de permanência na planta certamente favorece a dispersão. Esse comportamento de engolir os frutos foi apresentado principalmente pela família Tyrannidae, que também apresentou baixo tempo médio de permanência (0,88 min), o menor registrado 
entre todas as famílias de aves observadas no CCPIU, o que parece indicar que essa família pode ser importante para dispersão. Entretanto, comportamento de mandibulação do fruto pode prejudicar o processo de dispersão, pois a semente pode ser derrubada ou descartada sob a planta-mãe (SCHUPP, 1993), como foi observado na família Thraupidae no CCPIU e também por Moermond e Denslow (1985) e Borges e Melo (2012).

\section{CONCLUSÕES}

Este trabalho demonstrou a importância de duas espécies de plantas (Miconia theizans e Ouratea spectabilis) na rede de interações entre plantas e aves frugívoras apresentando elevado índice de importância de interações em área de Cerrado, no Triângulo Mineiro. Nossos dados sugerem que essas espécies podem ser importantes na recuperação de áreas degradadas não por serem espécies com elevado IVI fitossociológico (REIS; KAGEYAMA, 2008), mas por atraírem muitas aves dispersoras de sementes que podem carrear novas sementes para área, acelerando o processo de recuperação. Entre as espécies mais importantes no consumo de frutos, destacou-se Tyrannidae, do gênero Elaenia, que atuou como um dos principais dispersores de sementes em áreas abertas.

\section{AGRADECIMENTOS}

Ao Dr. J.N. Nakajima e à Dra. A. A. Arantes, pela identificação das plantas; à FAPEMIG, pela concessão da bolsa de iniciação científica (Projeto números 028/ 2000 e 080/2001); e à Diretoria do Clube Caça e Pesca Itororó e seus funcionários, pela autorização e pelo apoio ao estudo.

\section{REFERÊNCIAS}

ALMEIDA-NETO, M. et al. Vertebrate dispersal syndromes along the Atlantic forest: broad-scale patterns and macroecological correlates. Global Ecology and Biogeography, v.17, n.4, p.503-513, 2008.

AMÂNCIO, S.; MELO, C. Frugivoria por aves em bordas de fragmentos florestais, Uberlandia, MG. Horizonte Científico, v.1, p.1-19, 2008.

APPOLINÁRIO, V.; SCHIAVINI, I. Levantamento fitossociológico de espécies arbóreas de cerrado (stricto sensu) em Uberlândia - Minas Gerais.

Boletim do Herbário Ezechias Paulo Heringer, v.10, p.57-75, 1992.

Revista Árvore, Viçosa-MG, v.38, n.3, p.433-442, 2014
ATHIÊ, S.; DIAS, M. M. Frugivoria por aves em um mosaico de Floresta Estacional e reflorestamento misto em Rio Claro, São Paulo, Brasil. Acta Botanica Brasilica, v.26, n.1, p.84-93, 2012.

BATALHA, M. A.; ARAGAKI, S.; MANTOVANI, W. Variações fenológicas das espécies do cerrado em Emas (Pirassununga, SP). Acta Botanica Brasilica, v.11, n.1, p.61-78, 1997.

BORGES, M. R.; MELO, C. Frugivory and seed dispersal of Miconia theaezans (Bonpl.) Cogniaux (Melastomataceae) by birds in a transitions palm swamp - gallery forest in Central Brazil. Brazilian Journal of Biology, v.72, n.1, p.25-31, 2012.

CARVALHO, F. A. et al. Composição florística, riqueza e diversidade de um cerrado sensu stricto no sudeste do estado de Goiás. Bioscience Journal, v.24, n.1, p.64-72, 2008.

CAVALCANTI, R. B.; MARINI, M. A. Body masses of birds of the cerrado region, Brazil. Bulletin of the British Ornithologists' Club, v.113, p.209-212, 1993.

\section{COMITÊ BRASILEIRO DE REGISTROS}

ORNITOLÓGICOS - CBRO. Listas das aves do Brasil. 11.ed. 11 de jan. de 2014. Disponível em <http://www.cbro.org.br>. Acesso em: 23 de jul. de 2014.

FADINI, R. F.; MARCO JR., P. Interações entre aves frugívoras e plantas em um fragmento de mata atlântica de Minas Gerais. Ararajuba, v.12, n.1, p.15-21, 2004.

FAUSTINO, T. C.; MACHADO, C. G. Frugivoria por aves em uma área de campo rupestre na Chapada Diamantina, BA. Revista Brasileira de Ornitologia, v.14, n.1, p.137-143, 2006.

FELFILI, J. M. et al. Estudo fenológico de Stryphnodendron adstringens (Mart.) Coville no cerrado sensu stricto da Fazenda Água Limpa no Distrito Federal, Brasil. Revista Brasileira de Botânica, v.22, n.1, p.83-90, 1999.

FONSECA, F. Y.; ANTUNES, A. Z. Frugivoria e predação de sementes por aves no Parque Estadual Alberto Lofgren, São Paulo, SP. Revista do Instituto Florestal, v.19, n.1, p.81-91, 2007. 
FOSTER, M. S. Feeding methods and efficiencies of selected frugivorous birds. Condor, v.89, p.566-580, 1987.

GALETTI, M.; PIZO, M. A. Fruit eating by birds in a forest fragment in southeastern Brazil.

Ararajuba, v.4, n.1, p.71-79, 1996.

GALETTI, M.; STOTZ, D. Miconia hypoleuca (Melastomataceae) como espécie chave para aves frugívoras no Sudeste do Brasil. Revista Brasileira de Biologia, v.56, n.2, p.435-439, 1996.

GARCIA L. C.; BARROS, F. V.; LEMOS-FILHO, J. P. Fructification phenology as an important tool in the recovery of iron mining areas in Minas Gerais, Brazil. Brazilian Journal of Biology, v.69, n.3, p.887-893, 2009.

GOTTSBERGER, G.; SILLBERBAUER GOTTSBERGER, I. Dispersal and distribution in cerrado vegetation of Brazil. Sundered. Naturwiss Ver. Hamburg, v.7, p.313-352, 1983.

GRESSLER, E.; PIZO, M. A.; MORELLATO, L. P. C. Polinização e dispersão de sementes em Myrtaceae do Brasil. Revista Brasileira de Botanica, v.29, n.4, p.509-530, 2006.

JANZEN, D. H. Synchronization of sexual reproduction of trees within the dry season in Central America. Evolution, v.21, n.3, p.620$637,1967$.

JESUS, S.; MONTEIRO-FILHO, E.L.A. Frugivoria por aves em Schinus terebinthifolius (Anacardiaceae) e Myrsine coriacea (Myrsinaceae). Revista Brasileira de Ornitologia, v.15, n.4, p.585-591, 2007.

JORDANO, P. Fruits and frugivory. In: FENNER, M. (Ed.). Seeds: the ecology of regeneration in plant communities. Wallingford: Commonwealth Agricultural Bureau International, 2000. p.105156.

JORDANO, P.; SCHUPP, E.W. Determinants of seed disperser effectiveness: the quantity component and patterns of seed rain for Prunus mahaleb. Ecological Monographs, v.70, n.4, p.591-615, 2000.
LOISELLE, B. A.; BLAKE, J. G. Dispersal of melastomataceae seeds by fruit-eating birds of tropical forest understory. Ecology, v.80, n.1, p.330-336, 1999.

LOISELLE, B. A.; BLAKE, J. G. Potencial consequences of extinction of frugivorous birds for shrubs of tropical wet forest. In: LEVEY, D.J.; SILVA, W.R.; GALLETI, M. (Ed.) Seed dispersal and frugivory: ecology, evolution and conservation. Wallingford: CABI International, 2002. p.397-406.

MANTOVANI, W.; MARTINS, F. R. Variações fenológicas das espécies do cerrado da Reserva Biológica de Moji Guaçu, Estado de São Paulo.

Revista Brasileira de Botânica, v.11, n.1, p.101-112, 1988.

MARINI, M. A.; CAVALCANTI, R. B. Frugivory by Elaenia flycatchers. Hornero, v.15, n.1, p. 47-50, 1998.

MOERMOND, T. C.; DENSLOW, J. S. Neotropical avian frugivores: patterns of behavior, morphology, and nutrition with consequences for fruit selection. In: BUCKLEY, P. A. et al. (Ed.)

Neotropical Ornithological Monographs, v.36, p.865-897, 1985.

MORELLATO, P. C.; LEITÃO-FILHO, H. F. Padrões de frutificação e dispersão na Serra do Japi. In: MORELlATO, P. C. (Ed.) História Natural da Serra do Japi: ecologia e preservação de uma área florestal no sudeste do Brasil. Campinas: UNICAMP/FAPESP, 1992. p.112-140.

MOTTA-JUNIOR, J. C. Estrutura trófica e composição das avifaunas de três hábitats terrestres na região central do estado de São Paulo. Ararajuba, v.1, p.65-71, 1990.

MOTTA-JUNIOR, J. C.; LOMBARDI, J. A. Ocorrência de zoocoria em florestas-de-galeria no Complexo do Cerrado, Brasil. Biotemas, v.15, n.1, p.59-81, 2002.

PARRINI, R.; PACHECO, J. F. Frugivoria por aves em seis espécies arbóreas do gênero Miconia (Melastomataceae) na Mata Atlântica do Parque Nacional da Serra dos Órgãos, Região Sudeste do Brasil. Atualidades Ornitológicas, v.159, n.1, p.51-58, 2011.

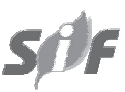

Revista Árvore, Viçosa-MG, v.38, n.3, p.433-442, 2014 
PEDRONI, F.; SANCHEZ, M.; SANTOS, F. A. M. Fenologia da copaíba (Copaifera langsdorffii Desf. - Leguminosae, Caesalpinioideae) em uma floresta semidecídua do sudeste do Brasil.

Revista Brasileira de Botânica, v.25, n.2, p.183-194, 2002.

PERES, C. A. Effects of subsistence hunting on vertebrate community structure in amazonian forests. Conservation Biology, v.14, n.1, p.240-253, 2000.

PIRANI, F. R.; SANCHEZ, M.; PEDRONI, F. Fenologia de uma comunidade arbórea em cerrado sentido restrito em Barra do Garças, MT. Acta Botanica Brasilica, v.23, n.4, p.1096-1109, 2009.

PRATT, T. K.; STILES, E.W. How long fruit-eating birds stay in the plants where they feed: implications for seed dispersal. American Naturalist, v.122, n.6, p.797-805, 1983.

REIS, A.; KAGEYAMA, P. Y. Restauração de áreas degradadas utilizando interações interespecíficas. In: KAGEYAMA, P. Y. et al. (Ed.). Restauração ecológica de ecossistemas naturais. Botucatu: FEPAF, 2008. p.91-110.

RIBEIRO, M. N. et al. Fogo e dinâmica da comunidade lenhosa em cerrado sentido restrito, Barra do Garças, Mato Grosso. Acta Botanica Brasilica, v.26, p.237-251, 2012.

RODRIGUES, M. Spatial distribution and food utilization among tanagers in southeastern Brazil (Passeriformes: Emberizidae). Ararajuba, v.3, n.1, p.27-32, 1995.

ROSA, R.; LIMA, S. C.; ASSUNÇÃO, W. L. Abordagem preliminar das condições climáticas de Uberlândia, MG. Sociedade e Natureza, v.1, n.1, p.61-66, 1991.

SCHUPP, E. W. Quantity, quality and the effectiveness of seed dispersal by animals. Vegetatio, v.107-108, n.1, p.15-30, 1993.

SICK, H. Ornitologia brasileira, uma introdução. Rio de Janeiro: Nova Fronteira, 1997. 912p.
SILVA, I. A.; CIANCUARUSO, M. V.; BATALHA, M. A. Dispersal modes and fruiting periods in hyperseasonal and seasonal savannas, central Brazil. Revista Brasileira de Botânica, v.32, n,1, p.155-163, 2009.

SILVA, J. M. C.; TABARELLI, M. Tree species impoverishment and the future flora of the Atlantic Forest of northeast Brazil. Nature, v.404, p.72-74, 2000.

SILVA, W. R. et al. Patterns of fruit-frugivore interactions in two Atlantic forest bird communities of southeastern Brazil: Implications for conservation. In: LEVEY, D. J.; SILVA, W. R.; GALETTI, M. (Ed.) Seed dispersal and frugivory: ecology, evolution and conservation. Wallingford: CABI International, 2002. p.423-435.

SNOW, B.K.; SNOW, D. W. The feeding ecology of tanagers and honeycreepers in Trinidad. The Auk, v.88, p.291-322, 1971.

TERBORGH, J. W. Keystone plant resources in the tropical forests. In: SOULÉ, M. E. (Ed.). Conservation biology: the science of scarcity and diversity. Sinauer: 1986. p.330-344.

VIEIRA, D. L. M. et al. Síndromes de dispersão de espécies arbustivo-arbóreas em cerrado sensu stricto do Brasil Central e savanas amazônicas. Revista Brasileira de Botânica, v.25, n.2, p.215-220, 2002.

WHEELWRIGHT, N. T.; JANSON, C. H. Colors of fruit displays of bird-dispersed plants in two tropical forests. The American Naturalist, v.126, n.6, p.777-799, 1985.

WUNDERLE JR., J. M. The role of animal seed dispersal in accelerating native forest regeneration on degraded tropical lands. Forestry Ecology and Management, v.99, n.1/2, p.223-235, 1997.

WUTHERICH, D. et al. Seed dispersal in Palicourea rigida, a common treelet species from tropical savannas. Journal of Tropical Ecology, v.17, n.3, p.449-458, 2001. 